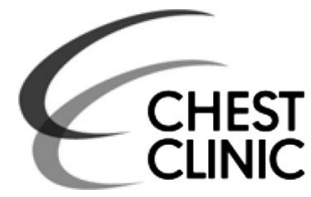

${ }^{1}$ Department of Respiratory and Critical Care Medicine, China-Japan Friendship Hospital, Beijing, China

${ }^{2}$ Department of Cardiovascular Medicine, China-Japan Friendship Hospital, Beijing, China

${ }^{3}$ Department of Respiratory Medicine, Capital Medical University, Beijing, China ${ }^{4}$ Beijing Key Laboratory of Respiratory and Pulmonary Circulation Disorders, Beijing, China

${ }^{5}$ National Clinical Research Center for Respiratory Diseases, Beijing, China

\section{Correspondence to} Dr Wanmu Xie, Department of Respiratory and Critical Care Medicine, China-Japan Friendship Hospital, 2 Yinghua Dongjie, ChaoYang District, Beijing 100029, China; xiewanmu@126.com

Received 17 June 2016 Revised 9 August 2016 Accepted 12 October 2016 Published Online First 4 November 2016

\title{
Metastatic synovial sarcoma of lung mimicking pulmonary embolism and deep venous thrombosis
}

\author{
Jiaojiao Qiu, ${ }^{1,2}$ Wanmu Xie, ${ }^{1,3,4}$ Zhenguo Zhai, ${ }^{1,3,4}$ Jun Wan, ${ }^{1,3,4}$ Chen Wang ${ }^{1,3,4,5}$
}

\section{INTRODUCTION}

Synovial sarcoma (SS) is a highly aggressive mesenchymal malignancy that usually originates from deep soft tissues of the extremities. Lungs are the most common metastatic site, but rare cases of vascular metastasis have been reported. Here we report a case of metastatic SS in a 26-year-old male patient with right lower extremity oedema first misdiagnosed as deep venous thrombosis (DVT). CT pulmonary angiogram (CTPA) revealed a mass lesion inside the right pulmonary artery mimicking PE. The diagnosis of SS was established by histopathological examination acquired by endobronchial ultrasound-guided transbronchial needle aspiration (EBUS-TBNA) from the right pulmonary artery. 18FDG-PET revealed that breviductor and pectineus muscle was the original site of SS with metastasis in common and external iliac veins, pulmonary artery and pulmonary parenchyma.

\section{CASE REPORT}

A 26-year-old man presented to our hospital with repeated right leg swelling and chest pain. Nine months before hospitalisation, the patient had right leg swelling and was diagnosed with DVT according to an ultrasound scan. Catheter-directed thrombolysis was performed followed by anticoagulation. The patient had slight relief in symptoms. Seven months later he had intermittent chest pain. CTPA (figure 1A) revealed intraluminal filling defects in the right pulmonary artery. He was diagnosed with PE and DVT, and took regular warfarin without any improvement. Then he was admitted to our hospital for further investigation. On general physical examination and routine blood tests, nothing was remarkable. A compressed ultrasound scan demonstrated thrombosis in the iliac, femoral superficial and great saphenous veins.
Our first diagnosis was PE and DVT based on clinical presentation and imaging results. But pulmonary artery sarcoma was suspected from CTPA, which showed mass lesions inside the pulmonary artery lumens, with multiple nodules dispersing at pulmonary lobes. Then EBUS-TBNA was performed and a specimen was taken from the right pulmonary artery (figure 1B). Histopathology revealed small cell malignancy, and immunohistochemistry (figure 2) showed a positive reaction for Vimentin and CD99. Cytogenetic study showed the presence of SS18(SYT) gene break-apart rearrangement on chromosome $18 \mathrm{q} 11$ by fluorescence in situ hybridisation, indicating a diagnosis of SS. To find the origin of the tumour, 18FDG-PET (figure 3) was performed, which showed dramatically increased FDG uptake in breviductor and pectineus muscle (SUVmax $=8)$, common and external iliac veins (SUVmax $=4.6-5.9$ ), the right lower pulmonary artery $(S U V m a x=4.1)$ and pulmonary nodules (SUVmax $=5.2$ ). Considering the histopathology, the breviductor and pectineus muscle were thought to be the primary site of SS. Thereafter, the patient received four cycles of chemotherapy with ifosfamide and doxorubicin and he was stable.

\section{DISCUSSION}

SS is a rare neoplasm mainly existing in soft tissues of the extremities. More than $75 \%$ of patients develop lung metastases within 1 year after presentation, most of which being parenchyma and pleural based, ${ }^{1}$ and pulmonary artery involvement is extremely rare. As far as we know, there are only two pulmonary artery metastasis cases reported in the literature, ${ }^{2}{ }^{3}$ but neither case had a history of lower extremity oedema. Our case had a clinical manifestation of unilateral leg swelling and chest pain, combined with a CTPA showing intraluminal filling defects in the right pulmonary artery
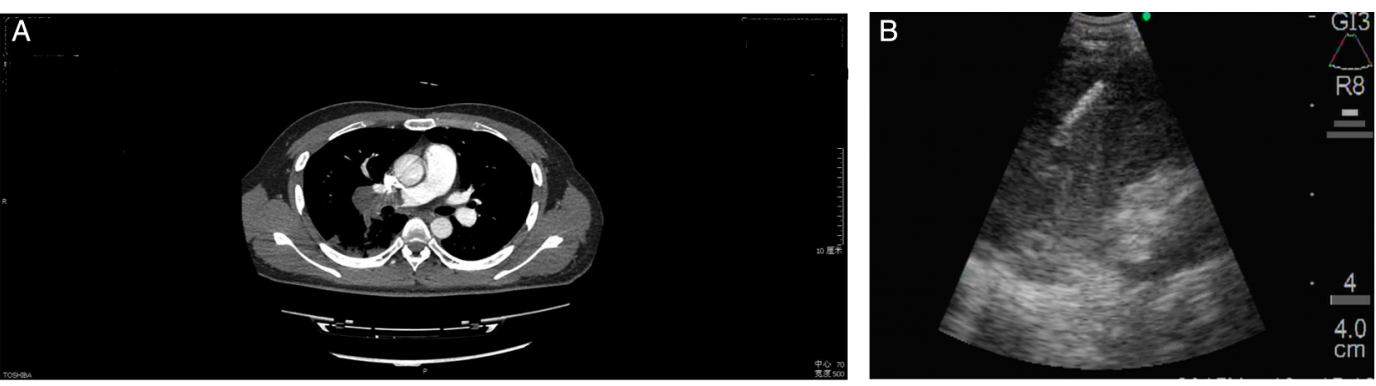

Figure 1 (A) CT pulmonary angiogram showing mass lesions inside right pulmonary artery lumen. (B) Endobronchial ultrasound-guided transbronchial needle aspiration (EBUS-TBNA) was performed for the mass-like lesion in the right pulmonary artery. 

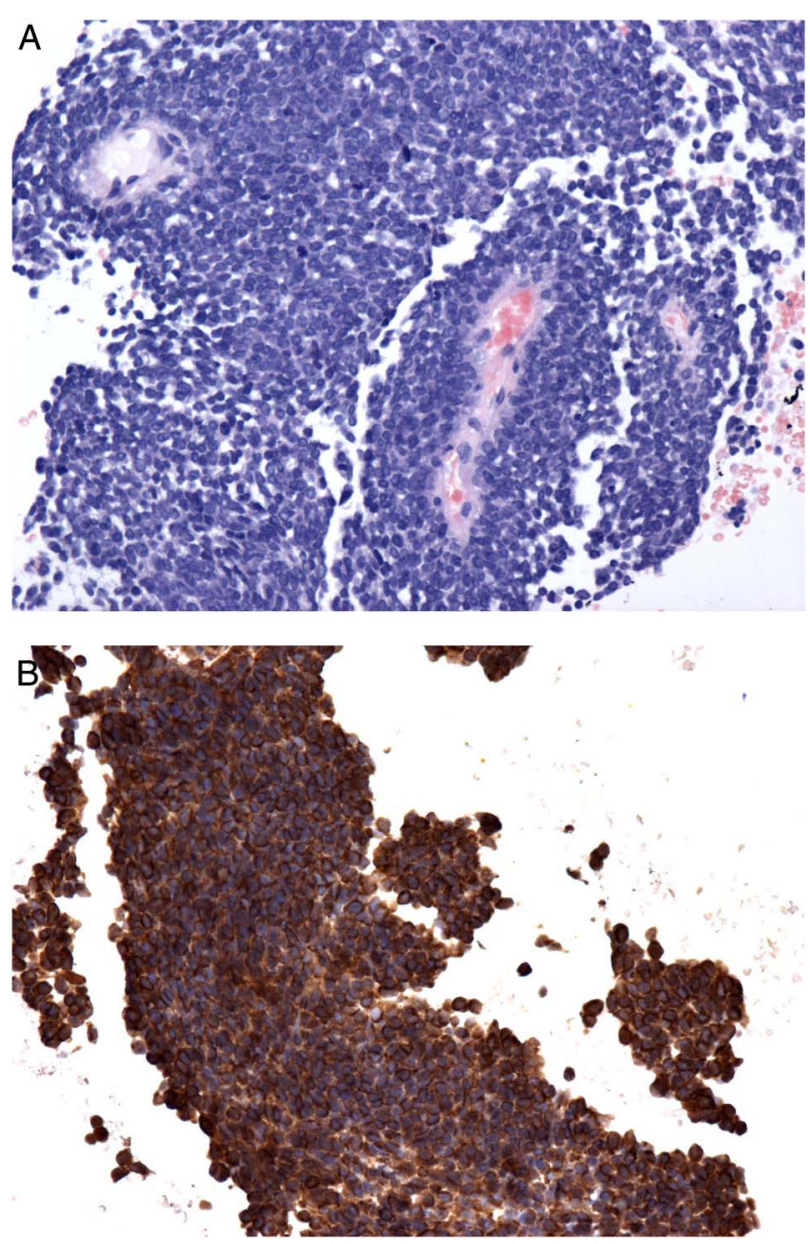

Figure 2 Microscopic study revealed a highly cellular tumour composed of relatively small, rounded cells $(A, H \& E)$. Immunohistochemical staining showed positive reaction for vimentin (B) $(\times 200)$. mimicking PE and DVT. Some clinical features alerted us to suspect malignancy. First, the patient did not improve with regular anticoagulation. Second, his imaging features, which showed mass lesions occupying the entire luminal diameter of the unilateral pulmonary artery, were not consistent with PE. These clues prompted us to investigate further.

To achieve a histopathological diagnosis, EBUS-TBNA was used successfully in this patient. Although the bleeding complications of this procedure have been questioned, we took into account that the mass was in direct contact with the pulmonary vessel wall as shown from the CT scan, and EBUS can detect the blood flow before it approaches the tissue. Therefore, this procedure may be considered safe as reported. ${ }^{45}$ It is difficult to make a definite diagnosis of SS even with histopathology. But immunohistochemistry and identification of SYT/SSX fusion transcript are useful for confirmation. ${ }^{6}$ Chemotherapy and radiotherapy are common therapy regimes for metastatic SS. The patient was given four cycles of ifosfamide and doxorubicin, which was regarded the first choice of chemotherapy. ${ }^{7} \mathrm{He}$ was stable at follow-up, but the long-term outcome is unclear.

In conclusion, pulmonary artery involvement of metastatic SS is rare and can be misdiagnosed as PE and DVT. Clinicians must consider metastatic sarcoma when making a differential diagnosis for patients with pulmonary artery filling defects.

Contributors JQ was a resident and wrote the first draft. WX, ZZ, JW and CW worked together and developed the therapy regimes and revised the manuscript. All authors had access to the data and played a role in writing this manuscript. WX is corresponding author.

Funding This work was supported by the National Key Technology R\&D Program of the Ministry of Science and Technology (2016YFC0905600, 2016YFC0905601, 2012BAI05B02), the National Natural Science Foundation of China (81570049) and the Beijing Natural Science Foundation (7152062).

Competing interests None declared.

Patient consent Obtained.

Provenance and peer review Not commissioned; externally peer reviewed.
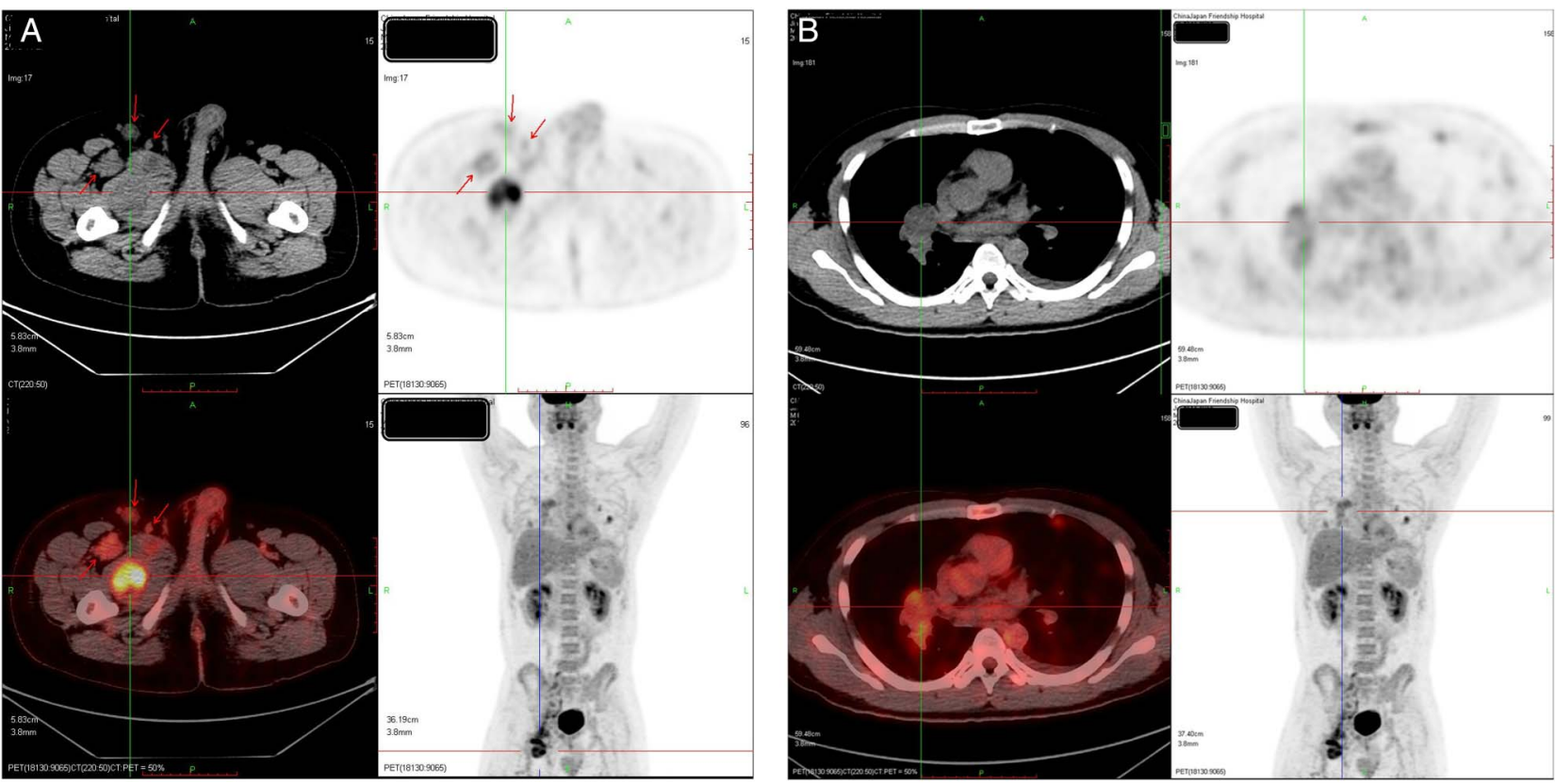

Figure 3 Representative images from 18FDG-PET showed increased FDG uptake in breviductor muscle $(A, S U V m a x=8)$ and the right lower pulmonary artery $(\mathrm{B}, \mathrm{SUVmax}=4.1)$. 


\section{REFERENCES}

1 Baheti $A D$, Tirumani $S H$, Sewatkar $R$, et al. Imaging features of primary and metastatic extremity synovial sarcoma: a single institute experience of 78 patients. Br J Radiol 2015;88:20140608.

2 Guo Q, Lu C, Guo Y. Pulmonary artery obstruction caused by a rare type of primary cardiac synovial sarcoma. J Thorac Cardiovasc Surg 2013;146:980.

3 Schmid S, Ohlschlegel C, Nagel W, et al. Pulmonary embolism in a patient with primary renal synovial sarcoma: the important differential diagnosis of tumor embolism and its therapeutic implications. Case Rep Oncol 2013;6: 331-8.
4 Park JS, Chung JH, Jheon S, et al. EBUS-TBNA in the differential diagnosis of pulmonary artery sarcoma and thromboembolism. Eur Respir J 2012;39:1480-2.

5 Dusemund F, Schneider T, Zeisel C, et al. Endobronchial ultrasound-guided transbronchial needle aspiration of an intravascular sarcoma metastasis. Respiration 2013;86:430-2.

6 Ren T, Lu Q, Guo W, et al. The clinical implication of SS18-SSX fusion gene in synovial sarcoma. Br J Cancer 2013;109:2279-85.

7 Tanaka K, Mizusawa J, Fukuda $\mathrm{H}$, et al. Perioperative chemotherapy with ifosfamide and doxorubicin for high-grade soft tissue sarcomas in the extremities (JCOG0304). Jpn J Clin Oncol 2015;45:555. 